\title{
Properties of LMC Planetary Nebulae and Parent Populations in the MACHO Database
}

D. R. Alves, C. Alcock, R. A. Allsman, T. S. Axelrod, A. C. Becker, D. P. Bennett, K. H. Cook, K. C. Freeman, K. Griest, J. Guern, M. J. Lehner, S. L. Marshall, D. Minniti, B. A. Peterson, P. J. Quinn, M. R. Pratt, A. W. Rodgers, C. W. Stubbs, W. Sutherland, D. Welch

This document was prepared for submittal to IAU 180th Symposium, International Astronomical Union,

Groningen, The Netherlands; August 26-30, 1996

October 31, 1996

This is a preprint of a paper intended for publication in a journal or proceedings. Since changes may be made before publication, this preprint is made available with the understanding that it will not be cited or reproduced without the permission of the author. 


\section{DISCLAIMER}

This document was prepared as an account of work sponsored by an agency of the United States Government. Neither the United States Government nor the University of California nor any of their employees, makes any warranty, express or implied, or assumes any legal liability or responsibility for the accuracy, completeness, or usefulness of any information, apparatus, product, or process

disclosed, or represents that its use would not infringe privately owned rights. Reference herein to any specific commercial product, process, or service by trade name, trademark, manufacturer, or otherwise, does not necessarily constitute or imply its endorsement, recommendation, or favoring by the United States Government or the University of California. The views and opinions of authors expressed herein do not necessarily state or reflect those of the United States Government or the University of California, and shall not be used for advertising or product endorsement purposes. 


\section{Properties of LMC Planetary Nebulae and Parent Populations in the MACHO Database}

D.R. Alves ${ }^{1}$, C. Alcock ${ }^{2}$, R.A. Allsman ${ }^{3}$, T.S Axelrod ${ }^{4}$, A.C Becker $^{6}$, D.P. Bennett ${ }^{7}$, K.H. Cook ${ }^{2}$, K.C. Freeman ${ }^{5}$, K. Griest ${ }^{8}$, J. Guern ${ }^{8}$, M.J. Lehner ${ }^{8}$, S.L. Marshall ${ }^{2}$, D. Minniti ${ }^{2}$, B.A. Peterson $^{5}$, P.J. Quinn ${ }^{9}$, M.R. Pratt ${ }^{6}$, A.W. Rodgers ${ }^{5}$, C.W. Stubbs $^{6}$, W. Sutherland ${ }^{10}$, D. Welch ${ }^{11}$,

${ }^{1}$ UC Davis/LLNL; ${ }^{2}$ LLNL; ${ }^{3}$ ANUSF; ${ }^{4}$ MSSSO/LLNL; ${ }^{5}$ MSSSO; ${ }^{6} \mathrm{CrPA} / \mathrm{U}$ Wash.; ${ }^{7}$ Notre Dame; ${ }^{8} \mathrm{CfPA} / \mathrm{UCSD} ;{ }^{9}$ ESO; ${ }^{10}$ Oxford; ${ }^{11}$ McMaster;

\section{Abstract}

The MACHO microlensing experiment's time-sampled photometry database contains blue and red lightcurves for nearly 9 million stars in the central bar region of the Large Magellanic Cloud (LMC). We have identified known LMC Planetary Nebulae (PN) in the database and find one, Jacoby 5 , to be variable. We additionally present data on the "parent populations" of LMC PN, and discuss the star formation history of the LMC bar.

The Variable LMC Planetary Nebula, Jacoby 5
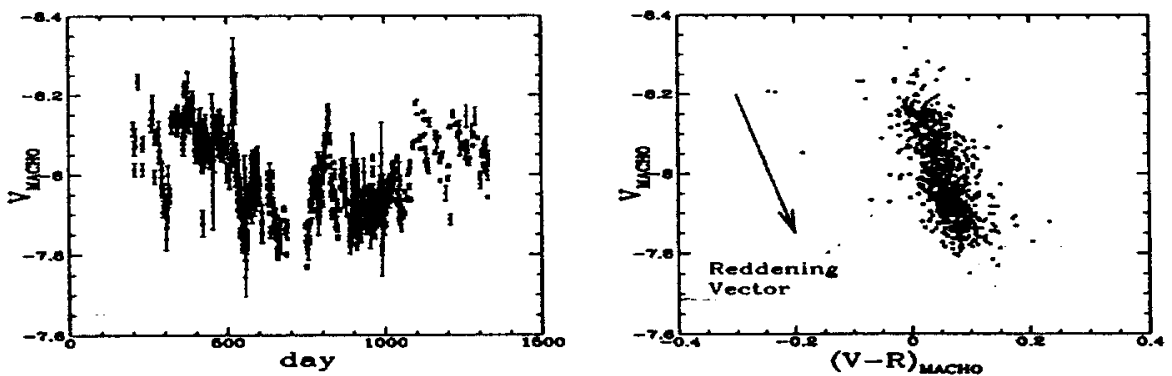

Fig. 1 - MACHO lightcurve for J5 starting Jan 2, 1992 and CMD

Fifty LMC PN from the catalogs of Jacoby [1] and SMP [2] have been identified in the MACHO database. One PN, Jacoby 5 (J5), is found to be variable on short timescales, of order tens of days, with an amplitude up to $0.4 \mathrm{mag}$ in the MACHO instrumental system [3]. The $V_{M A C H O}$ lightcurve and locus of points in the colormagnitude diagram (CMD) are presented in Fig. 1. Note that the points in the CMD lie parallel to the reddening vector. This may imply extinction as the source of variability. The point spread function of $\mathrm{J} 5$ is stellar. There is no correlation of brightness with seeing, and no periodicity is found. Boroson and Leibert [4] confirmed $\mathrm{J5}$ as a PN via spectrophotometric observations and remark on the presence of $\mathrm{Fe}$ emission lines. Broad-band photometric variability attributed to changes in emission line strengths has been seen in the galactic D-type symbiotic star, HM Sge [5]. Systems such as HM Sge are characterized by extremely high electron densities [6], and may be similar to J5. 


\section{Parent Populations}

MACHO has identified approximately 1500 Cepheids in the LMC bar [7]. Using photometry calibrated to $V$ and $R_{K C}$ and the pulsation models of Chiosi et al. [8] we derive a pulsation mass histogram ( $\mathrm{PMH}$ ) for metallicity appropriate to the LMC. Core He-burning "clump giants" have been counted with luminosity functions yielding $9.5 \times 10^{5}$ in the bar, or a surface density of 24 per $\operatorname{arcmin}^{2}$ in agreement with Hardy et al. [9].

We seek a "plausible" star formation history (SFH) constrained by the number of clump giants and Cepheid PMH. Our model uses the main sequence lifetimes of Iben and Laughlin [10], a Salpeter IMF, and a flat enrichment history. Cepheid lifetimes as a function of initial mass are derived from evolution tracks [11] and a theoretical instability strip [8]. We fit only the high mass end of the PMH. Clump lifetimes are derived from Vassilidias and Wood [12]. We count only stars of $M_{i}$ $\leq 2 M_{\odot}$ passing through the clump. We find that a constant $\mathrm{SFH}$ is improbable, as is a single ( $10 \mathrm{Gyr}$ ago) burst. Almost regardless of past $\mathrm{SFH}$, we find that a burst $50 \mathrm{Myr}$ ago $\left(\sigma_{F W H M}=50 \mathrm{Myr}\right.$ ) is required by the Cepheid PMH.

We estimate a total of $300 \pm 50 \mathrm{PN}$ in the bar of the LMC using observational data $[1,4]$ and predict only 150 for a lifetime of $2 \times 10^{4}$ years with our "plausible" present day mass function. Either the average LMC PN lifetime is disturbingly long $\left(4 \times 10^{4} \mathrm{yr}\right)$, the incompleteness of the surveys was overestimated, or a short duration burst of star formation took place a few Gyr ago. A modification to our SFH consistent with the Cepheid and clump giant constraints, favors the latter.

MACHO has also found 19,000 AGB red variables [3] where $87 \%$ have R-band amplitudes less than $0.5 \mathrm{mag}$, below the limits of previous surveys [13]. We find general agreement between our model predictions and the observed number of AGB variables, although pulsating lifetimes are highly uncertain [14]. We note that the gross disagreement between Mira deathrates and PN birthrates [13] is alleviated considerably by the large number of low amplitude AGB red variables found if they are all counted as "parents" of the LMC PN.

\section{REFERENCES}

[1] Jacoby, G. 1980 ApJS, 42, 1

[2] Sanduleak, N. et. al. 1978 PASP, 90, 621

[3] Cook, K.H., et. al. 1997 IAP Colloquium, ed. R. Ferlet (in press)

[4] Boroson, T. and Leibert, J. 1989 ApJ, 339, 844

[5] Ciatta, F. et. al. 1979 AA, 79, 247

[6] Schmid, H. and Schild, H. 1990 MNRAS, 246, 84

[7] Welch, D., et. al. 1997 IAP Colloquium, ed. R. Ferlet (in press)

[8] Chiosi, C. et. al. 1993 ApJS, 86, 541

[9] Hardy, E. et. al. 1984 ApJ, 278, 592

[10] Iben, I. and Laughlin, G. 1989 ApJ, 341, 312

[11] Castanelli, V. et. al. 1990 ApJS, 74, 463

[12] Vassilidas, E. and Wood, P. 1993 ApJ, 413, 641

[13] Hughes, S. and Wood. P. 1990 AJ, 99, 784

[14] Blocker, T. 1995 AA, 297, 727 


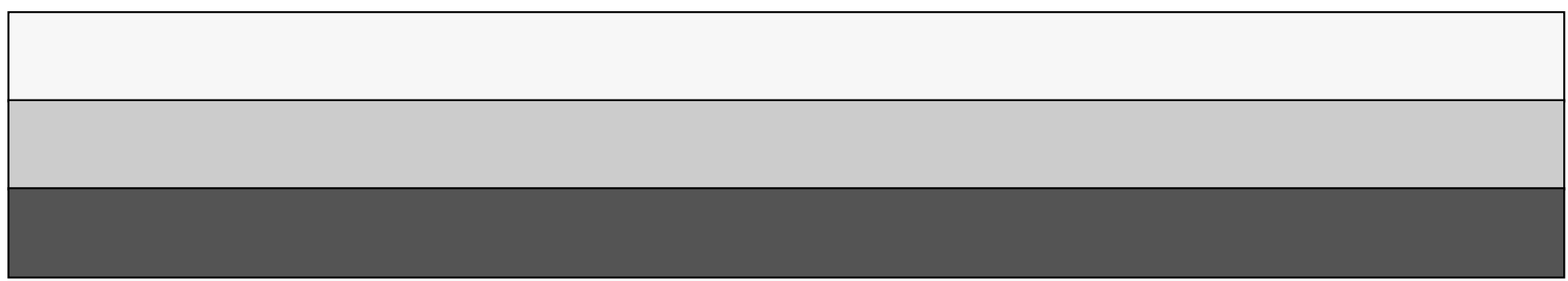

